\title{
Nonlinear metamaterial absorbers enabled by photonic doping of epsilon-near-zero metastructures
}

\author{
Ehsan Nahvi, ${ }^{1, *}$ Iñigo Liberal, ${ }^{1,2, \dagger}$ and Nader Engheta $\odot^{1, \hbar}$ \\ ${ }^{1}$ Department of Electrical and Systems Engineering, University of Pennsylvania, Philadelphia, Pennsylvania 19104, USA \\ ${ }^{2}$ Department of Electrical and Electronic Engineering, Public University of Navarra, Pamplona, Navarra 31006, Spain
}

(Received 16 January 2020; revised 3 April 2020; accepted 28 May 2020; published 2 July 2020)

\begin{abstract}
We theoretically demonstrate an approach for designing absorbers with strongly intensity-dependent absorption. The proposed absorbers consist of a spacer layer between a top resistive sheet and an underlying metallic substrate, akin to the traditional Salisbury screen, except for the use of an epsilon-near-zero slab with a nonlinear dielectric inclusion as the spacer layer. Such absorbers may be designed to exhibit highly tailorable absorption characteristics, including either saturable or reverse saturable absorption. In addition, the proposed nonlinear absorbers include interesting features such as high angular selectivity, insensitivity with respect to the absorber thickness, bandwidth tunability, and the possibility of operating with or without hysteresis. The proposed nonlinear absorbers may be appealing for several applications and nonlinear devices, such as optical limiters.
\end{abstract}

DOI: 10.1103/PhysRevB.102.035404

\section{INTRODUCTION}

Since the spiked interest in electromagnetic metamaterials from the turn of the century [1], epsilon-near-zero (ENZ) media have garnered significant attention due to their unique light-matter interaction characteristics [2], rendering them suitable for several novel applications, such as supercoupling [3-6], highly directive antennas [7,8], geometryinvariant resonant cavities [9], nonradiating modes in threedimensional (3D) cavities [10-13], and nonperturbative nonlinear optics [14-17]. Another interesting aspect of ENZ media is that they enable the design of metamaterials which circumvent the constraints imposed by conventional effective medium theories. For example, as a result of the vanishing permittivity, it has been shown that for transverse-magnetic (TM) polarization, the magnetic field distribution in a twodimensional (2D) ENZ becomes spatially uniform [18]. Exploiting this distinctive property, it has been demonstrated that the ENZ effective permeability may assume arbitrary values by embedding a single dielectric inclusion into the ENZ host, while maintaining its near-zero permittivity [19]. This concept is usually referred to as photonic doping, and it has been extended to nonlinear dielectric inclusions, demonstrating the possibility of obtaining an ENZ with a nonperturbative magnetic nonlinearity by using a dopant with a perturbative electric nonlinearity [20].

The most commonly used design for absorbers consists of a resistive sheet or patterned frequency-selective surface, on top of a dielectric spacer layer, backed by a metallic ground plane [21]. In some absorbers, such as the Salisbury

\footnotetext{
*nahvi@seas.upenn.edu

†inigo.liberal@unavarra.es

*engheta@ee.upenn.edu
}

screen [22] and Circuit Analog Absorbers [23], the absorption occurs in the thin sheet. In contrast, in other absorbers, such as the Dallenbach layer [24], the absorption occurs in the dielectric layer, instead. In fact, it has been shown that a lossy ENZ slab mounted on top of a ground plane may perfectly absorb an incident plane wave at a designable incidence angle [25].

The pioneering work by Landy et al. introducing the concept of perfect metamaterial absorbers [26], stimulated substantial research efforts for designing high performance absorbers [27], resulting in absorber designs exhibiting polarization-sensitive [28] or insensitive [29], wide-angle [30] or narrow-angle [31], narrowband [32] or broadband [33], and dynamically tunable characteristics [34], as well as designs with flexible substrates [30], spanning microwave [35], terahertz [36], and optical frequencies [37], for applications as diverse as photovoltaics [38], microbolometers [39], and refractive index sensors [40].

While the vast majority of studies on metamaterial absorbers have focused on linear designs, nonlinear absorbers which exhibit intensity-dependent absorption, i.e., either increasing or decreasing with incident intensity, may be desirable in certain contexts. However, relatively few nonlinear absorber designs have been proposed in the literature. These studies exploited the nonlinear change in permittivity either due to the thermal changes associated with the absorbed radiation [41] or arising from the Kerr effect [42,43]. At microwave frequencies, nonlinear absorbers were experimentally demonstrated by incorporating diodes, as nonlinear lumped elements, into metasurfaces [44,45]. More recently, the plasmonic parametric resonance was utilized for obtaining reverse saturable absorption [46]. In various frequency regimes, several nonlinear absorbers were proposed with distinctive characteristics. For instance, in the microwave regime, waveform-dependent [47] and electrically tunable [48] nonlinear metasurface absorbers were designed. Additionally, in 
(a)

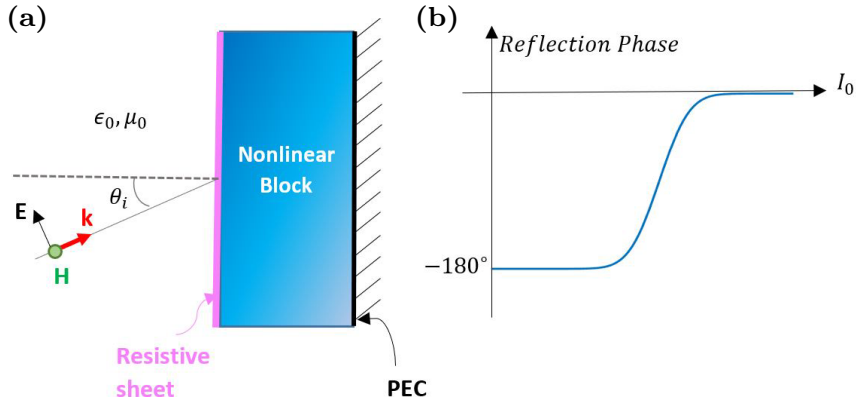

FIG. 1. Nonlinear absorbers from a general perspective. (a) Schematic of a plane-wave incident on a nonlinear absorber. (b) Quintessential nonlinear reflection phase variation. Reprinted with permission from our poster presentation at the 2020 CLEO, Ref. [63]. Copyright 2020 CLEO.

the terahertz regime, ultrathin, flexible nonlinear absorbers were proposed $[49,50]$.

In this article, we exploit the technique of nonlinear photonic doping to design absorbers which exhibit tailorable intensity-dependent absorption. As a result of its strong effective magnetic nonlinearity, the impedance of an ENZ slab backed by a Perfect Electric Conductor (PEC) varies considerably as the incident intensity increases. Consequently, the absorption spectrum shifts with increasing intensity. For a fixed frequency, however, the absorption varies considerably as a function of incident intensity.

\section{NONLINEAR ABSORBERS BASED ON PHOTONIC DOPING}

To explain the operating principle of the absorber, we start by revisiting the standard absorber configuration consisting of an absorptive sheet separated from a reflective ground plane with a spacer layer, one may envision achieving nonlinear absorption by deploying a nonlinear material in the spacer layer, as depicted schematically in Fig. 1(a). Assuming a reverse saturable absorption characteristic (i.e., low absorption for low incident intensities and high absorption for high intensities) is desired, the nonlinear spacer layer should behave such that the sheet becomes short-circuited at low incident intensities, whereas the impedance mismatch between the absorber and free-space decreases with increasing intensity.

In the absence of the resistive sheet, the reflection coefficient of a plane wave, obliquely incident from free-space, from a PEC-backed slab with permittivity $\epsilon_{1}$ and permeability $\mu_{1}$ may be readily shown to be given by the following expression by applying the continuity of the tangential electric and magnetic field components [51]:

$$
\Gamma=\frac{i \sqrt{\mu_{1}} \cos \left(\theta_{1}\right) \tan \left[k_{0} \sqrt{\mu_{1} \epsilon_{1}} \cos \left(\theta_{1}\right) d\right]+\sqrt{\epsilon_{1}} \cos \left(\theta_{1}\right)}{i \sqrt{\mu_{1}} \cos \left(\theta_{1}\right) \tan \left[k_{0} \sqrt{\mu_{1} \epsilon_{1}} \cos \left(\theta_{1}\right) d\right]-\sqrt{\epsilon_{1}} \cos \left(\theta_{1}\right)},
$$

where $\cos \left(\theta_{1}\right)=\sqrt{1-\sin ^{2}\left(\theta_{1}\right)}=\sqrt{1-\sin ^{2}\left(\theta_{i}\right) / \mu_{1} \epsilon_{1}}, k_{0}=$ $2 \pi / \lambda_{0}$, with $d$ and $\lambda_{0}$ denoting the slab thickness and freespace wavelength of the incident plane wave, respectively.

If the material parameters are chosen such that $\Gamma=-1$, the total electric field on the air/slab interface becomes zero.
As a result, no power would be dissipated in a resistive sheet placed on the slab. On the other hand, when $\Gamma=1$, the air/slab interface becomes an antinode for the electric field. As a result of the enhanced electric field, the absorption in the sheet becomes maximized. Therefore, a reverse saturable absorption characteristic is obtained if the slab is designed such that the reflection phase is 180 degrees for low intensity, but approaches zero phase as the incident intensity increases. A conceptual sketch of such a reflection phase variation is illustrated in Fig. 1(b).

This phase response can be implemented by using an ENZ slab doped with nonlinear dielectric inclusions to control its effective permeability. Specifically, the parameters of the doped ENZ slab are chosen such that $\mu_{\text {eff }} \simeq 0$ for low-intensity incident fields. As a result, the external boundary of the ENZ slab is effectively short-circuited for smallamplitude normally incident plane waves. As the intensity increases, however, the effective permeability of the doped ENZ increases, leading to a high impedance value, simply due to a near-zero permittivity, and hence approaching a $\Gamma=1$ reflection coefficient. As a result, the sheet exhibits negligible absorption for low-intensity normally incident plane waves, but strong absorption for higher incident intensities.

The overall behavior of the slab can also be explained by using an equivalent circuit model. Specifically, consider a TM-polarized plane wave normally incident on the absorber consisting of a sheet with resistivity $R_{s}$, separated from a PEC plane by a magnetic ENZ slab with permittivity $\epsilon_{\mathrm{ENZ}} \simeq 0$ and permeability $\mu_{\mathrm{ENZ}}(\omega)$. Due to the spatially uniform magnetic field in the ENZ, it has been shown that the ENZ slab may be modeled with a series reactance $X(\omega)=$ $\omega \mu_{0} \mu_{\mathrm{ENZ}}(\omega) d$, where $d$ denotes the ENZ thickness and $\omega$ is the angular frequency of the incident radiation [52]. As a result, the impedance seen behind the sheet, $Z_{1}(\omega)$ is given by (using the $e^{-i \omega t}$ time convention): $Z_{1}(\omega)=-i X(\omega)=$ $-i \omega \mu_{0} \mu_{\mathrm{ENZ}}(\omega) d$, leading to an input impedance $Z_{\text {in }}(\omega)$ from the absorber given by:

$$
Z_{\text {in }}(\omega)=Z_{1}(\omega) \| R_{s}=\frac{-i R_{s} \omega \mu_{0} \mu_{\mathrm{ENZ}}(\omega) d}{R_{s}-i \omega \mu_{0} \mu_{\mathrm{ENZ}}(\omega) d} .
$$

According to the transmission line model depicted in Fig. 2(a), the reflection from the absorber is given by $r=$ $\frac{Z_{\text {in }}(\omega)-Z_{0}}{Z_{\text {in }}(\omega)+Z_{0}}$. Since the absorber is backed by a PEC ground plane, the transmission coefficient is zero. Therefore, the absorption is simply given by $A=1-|r|^{2}$.

From the preceding equations, it is evident that $\mu_{\mathrm{ENZ}}(\omega) \simeq$ 0 leads to zero absorption. On the other hand, when $\left|\mu_{\mathrm{ENZ}}(\omega)\right|$ is sufficiently large such that $\left|\omega \mu_{0} \mu_{\mathrm{ENZ}}(\omega) d\right| \gg R_{s}$, then $Z_{\text {in }}(\omega) \simeq R_{s}$. Under this condition, the normally incident plane wave is completely absorbed if the sheet resistivity is chosen to coincide with the free-space impedance, i.e., $R_{s}=Z_{0}$.

According to the discussion above, one may design an absorber with reverse saturable absorption if the ENZ permeability vanishes for small incident intensity, but monotonically increases concurrently with the incident intensity. As was shown recently, an ENZ medium doped with a Kerr dielectric inclusion exhibits a nonperturbative effective magnetic nonlinearity while its effective permittivity remains near 
(a)

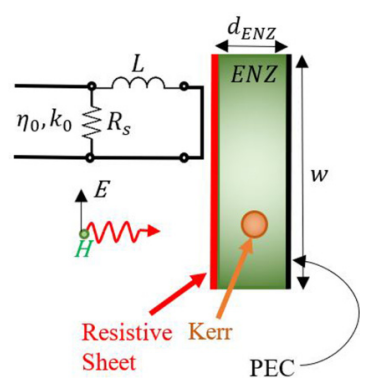

(b)

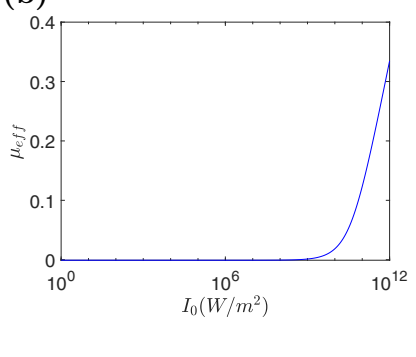

(c)

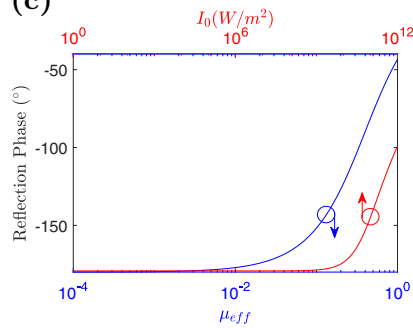

(d)

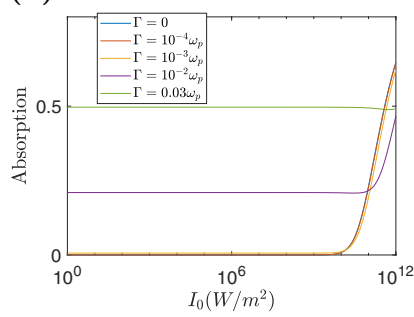

FIG. 2. A nonlinear absorber design, using a nonlinear doped ENZ. (a) Geometry of the nonlinear absorber, consisting of an ENZ with thickness $d_{\mathrm{ENZ}}=0.4 \lambda_{0}$ and periodicity $w=2.5 \lambda_{0}$ doped with a Kerr dielectric rod with radius $r_{d}=0.12307 \lambda_{0}$ and permittivity $\epsilon_{d}=$ $10+\frac{3 \chi^{(3)}}{\epsilon_{0}}|E|^{2}$, where $\chi^{(3)}=10^{-29}\left[\mathrm{C} . \mathrm{m} / \mathrm{V}^{3}\right]$. Inset: The transmission line model of the absorber. (b) Nonlinear effective permeability dependence on the intensity of a normally incident plane wave, in the absence of the resistive sheet. (c) Reflection phase as a function of incident intensity and relative permeability of the ENZ, in the absence of the resistive sheet. (d) The sheet absorption at $f=f_{p}$ for several representative levels of ENZ loss, as a function of incident intensity. Adapted with permission from our poster presentation at the 2020 CLEO, Ref. [63]. Copyright 2020 CLEO.

zero [20]. Therefore, as shown in Fig. 2(a), we consider deploying an ENZ slab doped with a Kerr dielectric rod, as the nonlinear block conceptualized earlier in Fig. 1(a).

Following the theory of photonic doping [19], the effective permeability description for a doped ENZ remains valid without any restrictions on the ENZ geometry and size. As a specific example, we consider an ENZ slab with thickness $d_{\mathrm{ENZ}}=0.4 \lambda_{0}$, periodically doped with periodicity $w=2.5 \lambda_{0}$ with a Kerr dielectric rod with permittivity $\epsilon_{d}=$ $10+\frac{3 \chi^{(3)}|E|^{2}}{\epsilon_{0}}$ where $\chi^{(3)}=10^{-29}\left(\mathrm{C} . \mathrm{m} / \mathrm{V}^{3}\right)$ [53]. To ensure that the effective permeability remains very small for lowintensity incident plane waves, we set the rod radius equal to $r_{d}=0.12307 \lambda_{0}$.

As the intensity of the wave incident on the PEC-backed doped ENZ increases, the effective permeability of the doped ENZ slab gradually starts to increase, in response to the Kerr effect in the dopant. As shown in Fig. 2(b), by integrating the magnetic field distributions in the ENZ and dopant [19], we obtain the effective permeability as a function of the intensity of a normally incident plane wave, in the absence of the resistive sheet. Also, it can be concluded from Fig. 2(b) that the effective permeability varies considerably with incident intensity, even though the permittivity of the dopant varies only in a perturbative fashion (see Fig. S1) [54].
In the absence of the resistive sheet, the reflection coefficient from the PEC-backed doped, lossless ENZ slab has a magnitude of unity. However, a variation in the effective permeability translates into a change in the reflection phase, as depicted in Fig. 2(c), following the general behavior illustrated in Fig. 1(b).

Having confirmed that the doped ENZ exhibits sufficiently strong nonlinearity to yield a considerable variation in the reflection phase, we now place a sheet with resistivity $R_{s}=$ $1 / \sigma d=\eta_{0}$ on top of the ENZ, where $\sigma$ and $d$ denote the sheet conductivity and thickness, respectively. Illuminating the absorber with a normally incident plane wave, we find that the sheet absorption indeed strongly depends on the incident intensity, as shown in Fig. 2(d). For low intensities, $\mu_{\text {eff }}(\omega) \simeq$ 0 , leading to negligible absorption. On the other hand, the absorption remarkably increases for higher intensities, due to the increased $\mu_{\text {eff }}(\omega)$.

Since all practical ENZ realizations inevitably have a nonzero amount of material loss, we also simulated for the nonlinear absorption characteristics assuming several representative levels of ENZ loss [55]. To quantify the ENZ loss, we assume the ENZ permittivity is given by a lossy Drude model where the loss is characterized by the collision frequency $\Gamma$. In the presence of ENZ loss, the dielectric loss in the ENZ adds with the Ohmic dissipation in the resistive sheet to account for the total absorption. For applications, such as solar cells [56], modulated thermal emitters [57,58], and optoelectronic devices [59], the absorption in the sheet is the main quantity of interest, whereas for other applications, such as reducing the radar cross section, the total absorption is the more significant variable. As shown in Fig. 2(d), the sheet absorption characteristic becomes less nonlinear as the ENZ becomes more lossy; however, the nonlinearity of the absorption remains substantial for moderately small ENZ loss. For comparison, the total absorption is plotted in Fig. S2 for the same representative levels of ENZ loss. We note that the geometry of the system could be optimized to work for a given amount of loss. For example, it is possible to reduce the sensitivity with respect to ENZ loss by decreasing the ENZ periodicity, albeit at the cost of a reduced nonlinearity (see Fig. S3).

Different classes of nonlinear absorbers could be designed by using this methodology. For example, in contrast to the reverse saturable absorption characteristic obtained when the sheet is placed on top of the ENZ, by placing the resistive sheet a quarter-wavelength away from the ENZ slab, a saturable absorption characteristic may be achieved where the absorption gradually decreases from total absorption at low intensities to smaller absorption at higher incident intensities (see Fig. S4). In the absence of the sheet, the reflection has a zero phase when the ENZ effective permeability is near zero, assuming the reference plane located a quarter-wavelength from the ENZ slab. An increase in the ENZ effective permeability, however, leads to an increase in the reflection phase. Assuming the ENZ is doped with a Kerr dielectric dopant, the effective permeability increases in response to higher incident intensity, leading to a reflection phase depending on the incident intensity. Therefore, in the presence of the resistive sheet, a nonlinear saturable absorption characteristic is obtained. 

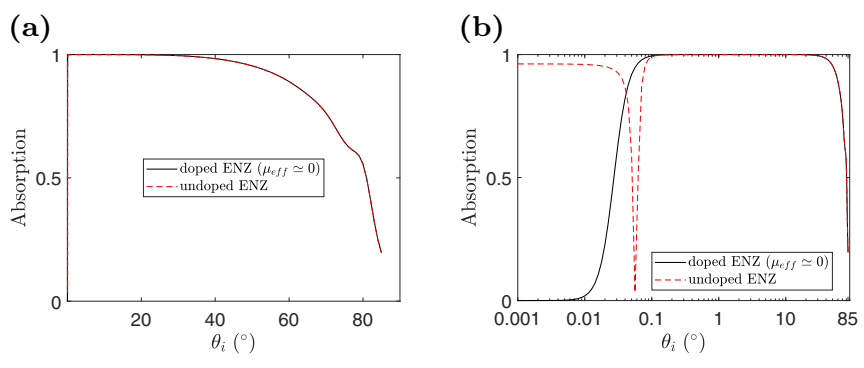

FIG. 3. The absorption of a plane wave obliquely incident on the absorber with the incidence angle shown on a (a) linear and (b) logarithmic scale. Reprinted with permission from our poster presentation at the 2020 CLEO, Ref. [63]. Copyright 2020 CLEO.

\section{GEOMETRICAL, ANGULAR AND FREQUENCY CHARACTERISTICS OF NONLINEAR ABSORBERS BASED ON PHOTONIC DOPING}

In contrast with most electromagnetic absorbers (e.g., Salisbury screen, Circuit Analog Absorbers, and Dallenbach layer), the operation principle of our nonlinear absorber does not critically depend on its thickness. This is illustrated in the absorber presented in Fig. 2, where we arbitrarily assumed an ENZ slab with thickness $d_{\mathrm{ENZ}}=0.4 \lambda_{0}$. However, the ENZ may also be designed to be much thinner compared to the wavelength. For instance, it would be possible to use thin rectangular dopants directly in contact with the PEC ground plane (see Fig. S5). In this manner, it is possible to drastically reduce the overall thickness of the absorber while maintaining the same operation principle. Therefore, the proposed configuration provides a greater geometrical flexibility and insensitivity with respect to the absorber thickness than other electromagnetic absorbers.

The angular response of the proposed absorber also exhibits some unique characteristics. In particular, in the absence of the resistive sheet, for a TM-polarized plane wave obliquely incident on a PEC-backed slab of permittivity $\epsilon_{1} \simeq 0$ and permeability $\mu_{1}$ with angle $\theta_{i}$, such that $\sin \left(\theta_{i}\right)>\mu_{1} \epsilon_{1}$, we have

$$
\cos \left(\theta_{1}\right)=\sqrt{1-\frac{\sin ^{2}\left(\theta_{i}\right)}{\mu_{1} \epsilon_{1}}}= \pm i \sqrt{\frac{\sin ^{2}\left(\theta_{i}\right)}{\mu_{1} \epsilon_{1}}-1} .
$$

Assuming the ENZ permittivity is sufficiently near zero, for an incidence angle $\theta_{i} \neq 0$, we have $\sin ^{2}\left(\theta_{i}\right) \gg \mu_{1} \epsilon_{1}$. Therefore $\cos \left(\theta_{1}\right) \simeq \pm i \frac{\sin \left(\theta_{i}\right)}{\sqrt{\mu_{1} \epsilon_{1}}}$.

Substituting Eq. (3) into Eq. (1), we obtain

$$
\Gamma \simeq \frac{-i \sin \left(\theta_{i}\right) \tanh \left[k_{0} \sin \left(\theta_{i}\right) d\right]+\epsilon_{1} \cos \left(\theta_{i}\right)}{-i \sin \left(\theta_{i}\right) \tanh \left[k_{0} \sin \left(\theta_{i}\right) d\right]-\epsilon_{1} \cos \left(\theta_{i}\right)} .
$$

From Eq. (4), it can be readily seen that the reflection coefficient from a PEC-backed ENZ becomes essentially independent of its permeability for oblique incidence angles which satisfy the condition $\sin ^{2}\left(\theta_{i}\right) \gg \mu_{1} \epsilon_{1}$. In Fig. 3, assuming $\epsilon_{1}=10^{-6}$, for a doped ENZ with $\mu_{\text {eff }}(\omega) \simeq 0$ and an undoped ENZ with $\mu_{1}=1$, we plot the absorption dependence on the incidence angle, with $\theta_{i}$ plotted on a linear and logarithmic scale. Our full-wave simulations corroborate that the absorption becomes virtually independent of the ENZ

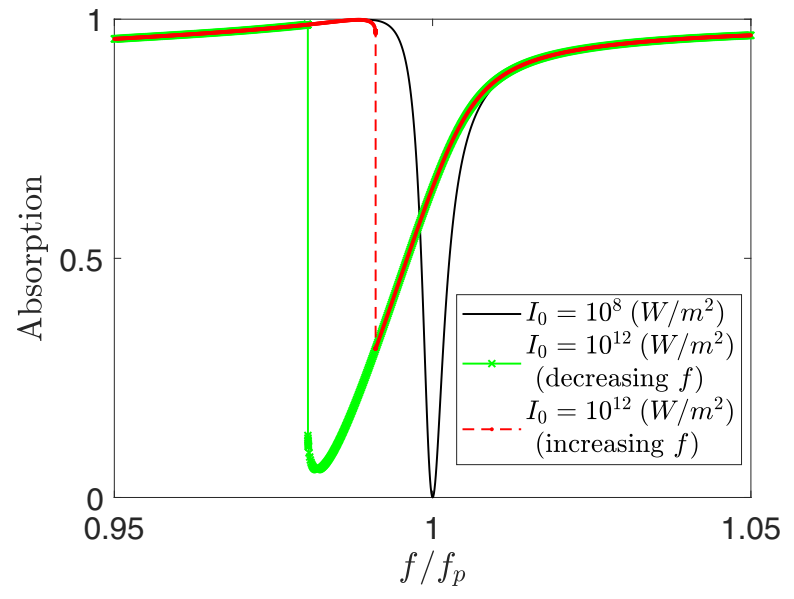

FIG. 4. The absorption spectra at normal incidence for low and high incident intensities, corresponding to $I_{0}=10^{8}\left(\mathrm{~W} / \mathrm{m}^{2}\right)$ and $I_{0}=10^{12}\left(\mathrm{~W} / \mathrm{m}^{2}\right)$, respectively. Reprinted with permission from our poster presentation at the 2020 CLEO, Ref. [63]. Copyright 2020 CLEO.

permeability. This effect can also be understood by noting that a body with simultaneously near-zero permittivity and permeability conforms a DB boundary [60], where both the normal D and B fields must be zero. This boundary presents a very high reflection at all angles except normal incidence, and, therefore, the proposed nonlinear absorber exhibits an extremely angular-sensitive response.

The radius of the dielectric rod inclusion has been selected such that $\mu_{\text {eff }} \simeq 0$ for low intensities at the ENZ plasma frequency $f=f_{p}$. However, due to the strong sensitivity of the ENZ effective permeability to the dopant's electrical size, the effective permeability is strongly dispersive, implying that the $\mu_{\text {eff }}(\omega) \simeq 0$ condition may hold for only a very narrow frequency range, smaller than the ENZ bandwdith of the host. As shown in Fig. 4, the dispersive effective permeability translates to a strongly frequency-selective absorption spectrum. For low incident intensities, the absorption is negligible only in a narrow bandwidth around $f_{p}$ where the effective permeability vanishes. As the intensity of the normally incident plane wave increases, the frequency for which the $\mu_{\text {eff }}(\omega) \simeq$ 0 condition holds shifts to a lower frequency, leading to a redshift of the absorption spectrum.

For high intensities, however, as a result of the nonlinear effects becoming prominent, we found the absorption spectrum to exhibit hysteresis and bistability, qualitatively similar to the hysteresis theoretically predicted while varying the frequency in a metamaterial with conformational nonlinearity [61] and experimentally obtained from a light-controlled split-ring resonator [62]. The bistability is observed near the resonance corresponding to $\left|\mu_{\text {eff }}\right| \rightarrow \infty$. However, since such an undesirable resonance is not reached as the intensity of a normally incident plane wave with frequency $f_{p}$ increases, we did not obtain hysteresis and bistability in the intensitydependent absorption characteristic presented in Fig. 2(d). Therefore, the proposed absorber enables operation both with and without hysteresis, depending on whether the system is designed to cross through the dopant resonance. 
Deploying linear dielectrics instead of the Kerr inclusions, one may realize highly tailorable linear absorbers where the absorption bandwidth may be tailored, in addition to dynamically shifting the absorption spectrum. For example, the absorption characteristics may become continuously more narrowband with a choice of a larger periodicity for the doped ENZ, modifying the rod radius to maintain the $\mu_{\text {eff }}(\omega) \simeq 0$ condition for low intensities (see Fig. S6). Moreover, the central frequency of the absorption resonance could be dynamically shifted by tuning the permittivity of the dielectric dopant (see Fig. S7). Therefore, doped ENZ slabs may also constitute the key building block in linear absorbers with highly tailorable absorption spectra.

\section{CONCLUSIONS}

In conclusion, we proposed and theoretically studied an approach for designing nonlinear absorbers which exhibit tailorable intensity-dependent absorption characteristics. Our design exploits the significantly enhanced nonlinearity of the recently introduced nonlinear doped ENZ structures. In addition, the absorption characteristics may be highly tailored by either judiciously selecting the dimensions of the ENZ host or by dynamically tuning the dopant permittivity. Furthermore, as an extra degree of flexibility, the absorbers may be designed to exhibit either saturable or reverse saturable absorption. We envision these nonlinear absorbers being beneficial for several applications, such as optical limiters.

\section{ACKNOWLEDGMENTS}

The authors acknowledge support, in part, from the US Air Force Office of Scientific Research (AFOSR) Multidisciplinary University Research Initiative (MURI) Grant No. FA9550-14-1-0389, and, in part, from the Vannevar Bush Faculty Fellowship program sponsored by the Basic Research Office of the Assistant Secretary of Defense for Research and Engineering and funded by the Office of Naval Research through Grant No. N00014-16-1-2029. Portions of this work were also presented as a poster at the CLEO 2020, see Ref. [63].
[1] J. B. Pendry, Phys. Rev. Lett. 85, 3966 (2000).

[2] I. Liberal and N. Engheta, Nat. Photon. 11, 149 (2017).

[3] M. Silveirinha and N. Engheta, Phys. Rev. Lett. 97, 157403 (2006).

[4] M. G. Silveirinha and N. Engheta, Phys. Rev. B 76, 245109 (2007).

[5] B. Edwards, A. Alù, M. E. Young, M. Silveirinha, and N. Engheta, Phys. Rev. Lett. 100, 033903 (2008).

[6] B. Edwards, A. Alù, M. G. Silveirinha, and N. Engheta, J. Appl. Phys. 105, 044905 (2009).

[7] S. Enoch, G. Tayeb, P. Sabouroux, N. Guérin, and P. Vincent, Phys. Rev. Lett. 89, 213902 (2002).

[8] R. W. Ziolkowski, Phys. Rev. E 70, 046608 (2004).

[9] I. Liberal, A. M. Mahmoud, and N. Engheta, Nat. Commun. 7, 10989 (2016).

[10] M. G. Silveirinha, Phys. Rev. A 89, 023813 (2014).

[11] F. Monticone and A. Alù, Phys. Rev. Lett. 112, 213903 (2014).

[12] I. Liberal and N. Engheta, Sci. Adv. 2, e1600987 (2016).

[13] F. Monticone, H. M. Doeleman, W. Den Hollander, A. F. Koenderink, and A. Alù, Laser Photonics Rev. 12, 1700220 (2018)

[14] C. Argyropoulos, P.-Y. Chen, G. D’Aguanno, N. Engheta, and A. Alu, Phys. Rev. B 85, 045129 (2012).

[15] A. Capretti, Y. Wang, N. Engheta, and L. Dal Negro, Opt. Lett. 40, 1500 (2015).

[16] M. Z. Alam, I. De Leon, and R. W. Boyd, Science 352, 795 (2016).

[17] L. Caspani, R. P. M. Kaipurath, M. Clerici, M. Ferrera, T. Roger, J. Kim, N. Kinsey, M. Pietrzyk, A. DiFalco, V. M. Shalaev, A. Boltasseva, and D. Faccio, Phys. Rev. Lett. 116, 233901 (2016).

[18] M. Silveirinha and N. Engheta, Phys. Rev. B 75, 075119 (2007).

[19] I. Liberal, A. M. Mahmoud, Y. Li, B. Edwards, and N. Engheta, Science 355, 1058 (2017).

[20] E. Nahvi, I. Liberal, and N. Engheta, ACS Photonics 6, 2823 (2019).
[21] C. M. Watts, X. Liu, and W. J. Padilla, Adv. Materials 24, OP98 (2012).

[22] W. W. Salisbury, Absorbent body for electromagnetic waves, US Patent No. 2,599,944 (10 June 1952).

[23] E. F. Knott, J. F. Schaeffer, and M. T. Tulley, Radar Cross Section (SciTech Publishing, Raleigh, NC, 2004).

[24] W. Dallenbach and W. Kleinsteuber, Hochfreq. Elektroak 51, 152 (1938).

[25] S. Zhong, Y. Ma, and S. He, Appl. Phys. Lett. 105, 023504 (2014).

[26] N. I. Landy, S. Sajuyigbe, J. J. Mock, D. R. Smith, and W. J. Padilla, Phys. Rev. Lett. 100, 207402 (2008).

[27] Y. Ra'Di, C. R. Simovski, and S. A. Tretyakov, Phys. Rev. Appl. 3, 037001 (2015).

[28] X. Xiong, Z.-H. Xue, C. Meng, S.-C. Jiang, Y.-H. Hu, R.-W. Peng, and M. Wang, Phys. Rev. B 88, 115105 (2013).

[29] N. I. Landy, C. M. Bingham, T. Tyler, N. Jokerst, D. R. Smith, and W. J. Padilla, Phys. Rev. B 79, 125104 (2009).

[30] H. Tao, C. M. Bingham, A. C. Strikwerda, D. Pilon, D. Shrekenhamer, N. I. Landy, K. Fan, X. Zhang, W. J. Padilla, and R. D. Averitt, Phys. Rev. B 78, 241103(R) (2008).

[31] C. H. Granier, F. O. Afzal, S. G. Lorenzo, M. Reyes, Jr., J. P. Dowling, and G. Veronis, J. Appl. Phys. 116, 243101 (2014).

[32] I. Puscasu and W. L. Schaich, Appl. Phys. Lett. 92, 233102 (2008).

[33] P. Yu, L. V. Besteiro, Y. Huang, J. Wu, L. Fu, H. H. Tan, C. Jagadish, G. P. Wiederrecht, A. O. Govorov, and Z. Wang, Adv. Opt. Mater. 7, 1800995 (2019).

[34] B. Zhu, C. Huang, Y. Feng, J. Zhao, and T. Jiang, Prog. Electromagnetics Res. 24, 121 (2010).

[35] S. Simms and V. Fusco, Electronics Letters 41, 1311 (2005).

[36] H. Tao, N. I. Landy, C. M. Bingham, X. Zhang, R. D. Averitt, and W. J. Padilla, Opt. Express 16, 7181 (2008).

[37] J. Hao, J. Wang, X. Liu, W. J. Padilla, L. Zhou, and M. Qiu, Appl. Phys. Lett. 96, 251104 (2010). 
[38] H. A. Atwater and A. Polman, Nature Materials 9, 205 (2010).

[39] T. Maier and H. Brückl, Optics Lett. 34, 3012 (2009).

[40] N. Liu, M. Mesch, T. Weiss, M. Hentschel, and H. Giessen, Nano Lett. 10, 2342 (2010).

[41] S. Guddala, R. Kumar, and S. A. Ramakrishna, Appl. Phys. Lett. 106, 111901 (2015).

[42] Y. Gong, Z. Li, J. Fu, Y. Chen, G. Wang, H. Lu, L. Wang, and X. Liu, Opt. Express 19, 10193 (2011).

[43] S. Guddala and S. A. Ramakrishna, Optics Lett. 41, 5150 (2016).

[44] H. Wakatsuchi, S. Kim, J. J. Rushton, and D. F. Sievenpiper, Appl. Phys. Lett. 102, 214103 (2013).

[45] S. Kim, H. Wakatsuchi, J. J. Rushton, and D. F. Sievenpiper, Appl. Phys. Lett. 108, 041903 (2016).

[46] S. Fardad and A. Salandrino, Optics Lett. 43, 6013 (2018).

[47] H. Wakatsuchi, S. Kim, J. J. Rushton, and D. F. Sievenpiper, Phys. Rev. Lett. 111, 245501 (2013).

[48] Z. Luo, J. Long, X. Chen, and D. Sievenpiper, Appl. Phys. Lett. 109, 071107 (2016).

[49] H. R. Seren, J. Zhang, G. R. Keiser, S. J. Maddox, X. Zhao, K. Fan, S. R. Bank, X. Zhang, and R. D. Averitt, Light: Science \& Applications 5, e16078 (2016).

[50] X. Zhao, J. Zhang, K. Fan, G. Duan, G. D. Metcalfe, M. Wraback, X. Zhang, and R. D. Averitt, Photonics Res. 4, A16 (2016).

[51] S. Zhong and S. He, Sci. Rep. 3, 2083 (2013).
[52] F. Abbasi and N. Engheta, Opt. Express 22, 25109 (2014).

[53] B. E. Saleh and M. C. Teich, Fundamentals of Photonics (John Wiley \& Sons, New York, 2019).

[54] See Supplemental Material at http://link.aps.org/supplemental/ 10.1103/PhysRevB.102.035404 for further results on the effect of ENZ loss, alternative nonlinear absorber designs, and tunable frequency spectra of linear absorbers.

[55] COMSOL Multiphysics® v. 5.4, www.comsol.com, COMSOL AB, Stockholm, Sweden.

[56] C. Hagglund and S. P. Apell, J. Phys. Chem. Lett. 3, 1275 (2012).

[57] J. Park, J.-H. Kang, X. Liu, S. J. Maddox, K. Tang, P. C. McIntyre, S. R. Bank, and M. L. Brongersma, Sci. Adv. 4, eaat3163 (2018).

[58] E. Sakat, L. Wojszvzyk, J.-P. Hugonin, M. Besbes, C. Sauvan, and J.-J. Greffet, Optica 5, 175 (2018).

[59] M. S. Jang, V. W. Brar, M. C. Sherrott, J. J. Lopez, L. Kim, S. Kim, M. Choi, and H. A. Atwater, Phys. Rev. B 90, 165409 (2014).

[60] I. V. Lindell and A. H. Sihvola, Phys. Rev. E 79, 026604 (2009).

[61] M. Lapine, I. V. Shadrivov, D. A. Powell, and Y. S. Kivshar, Sci. Rep. 1, 138 (2011).

[62] A. P. Slobozhanyuk, P. V. Kapitanova, I. Shadrivov, P. A. Belov, and Y. S. Kivshar, JETP Lett. 95, 613 (2012).

[63] E. Nahvi, I. Liberal, and N. Engheta, Design of nonlinear absorbers via doped ENZ metastructures, in 2020 Conference on Lasers and Electro-Optics (CLEO) (2020), pp. 1-2. 